\title{
Worsening of tree-related public health issues under climate change
}

To the Editor - With planting more trees being suggested as an effective way to combat climate change ${ }^{1,2}$, this seems to be a good time to emphasize other important public health issues related to trees. Planting trees can bring many benefits, including carbon sequestration, slowing surface water runoff, preventing soil erosion, providing shade and improving aesthetics. However, trees in the proximity of people may cause negative health implications that are further exaggerated by climate change, creating a potential double risk factor. These health aspects need to be considered when considering tree planting as a policy objective, as well as during implementation.

An important public health impact is the outbreak of oak processionary moth (OPM) caterpillars in the summer of 2019 across Europe, with the soaring number of complaints caused by their urticating hairs. OPM is considered one of the three major Lepidoptera species (together with pine processionary moths and brown-tail moths) in Europe whose caterpillars can cause symptoms such as malaise, urticaria, conjunctivitis, pharyngitis and respiratory distress in affected people ${ }^{3}$. One million microscopically small hairs on each OPM caterpillar can cause mechanical irritation of the skin and eyes, resulting in pseudo-allergic reactions referred to as lepidopterism, which has been defined as an airborne disease ${ }^{4}$. Lepidopterism usually goes underdiagnosed and underreported, but being mentioned as a 'plague' in the BBC News raised public awareness ${ }^{5}$. In addition to their direct effects (such as discomfort and medical conditions), OPM caterpillars may affect public health by causing negative emotions and reducing opportunities for, and enjoyment of, outdoor activities, thereby further increasing economic and chronic disease burdens ${ }^{6}$.

The outbreak of OPM caterpillars could, to some extent, be understood from a spatial life course epidemiology perspective if nature is imagined as an 'individual', with rising risks driven by a succession of milder winters and warmer springs as a result of climate change. The dramatic expansion of
OPMs in the Netherlands since 1989 ( ref. $^{8}$ ) could imply that such outbreaks are no coincidence: it may be just a beginning of more tree-related issues in warmer cities as a consequence of public policy favouring that cities are 'greened up' in order to reduce the impact of heat stress.

OPM caterpillars are not alone. Tree pollen is also an important cause of allergic reactions with remarkable clinical impacts; for example, ill-chosen tree species producing high airborne pollen concentration over an extended pollen season'. This has been observed in many Northern Hemisphere countries, including the USA and China, and could be exacerbated by climate change. A call for such efforts to improve long-term pollen monitoring has also been made among Southern Hemisphere countries given the increasing numbers of people with allergic rhinitis and cumulative abundance of pollen as well as an earlier onset of the tree pollen season ${ }^{10}$. Moreover, higher rates of tick invasions bearing diseases such as Lyme may be potentially worsened by tree planting, especially due to climate change or recent disturbances to forested areas that are attractive habitats for ticks and their hosts ${ }^{11}$. Often, however, simple public health actions can mitigate effects, such as planting the female individual of dioecious species, providing drainage and moving compost, and replacing vegetation preferred by ticks in (sub)urban settings.

Underreported diseases should not be neglected. Given the potential health effects of planting more trees in a changing climate, public health and climate researchers as well as policy-makers, non-governmental organizations, private sector actors and healthcare services must work together to produce evidence-based recommendations to mitigate health aspects of future treeplanting projects (that is, precision treeplanting), especially in urban areas where most people live.

\section{Peng Jia (D) 1,2,3凶, Tiejun Wang (D) 2四, Arnold J. H. van Vliet ${ }^{4}$, Andrew K. Skidmore ${ }^{2,5}$ and Maarten van Aalst ${ }^{2,6,7}$}

${ }^{1}$ International Initiative on Spatial Lifecourse Epidemiology (ISLE), Hong Kong, China. ${ }^{2}$ Faculty of Geo-Information Science and Earth Observation, University of Twente, Enschede, The Netherlands. ${ }^{3}$ Department of Land Surveying and Geo-Informatics, The Hong Kong Polytechnic University, Hong Kong, China. ${ }^{4}$ Environmental Systems Analysis Group, Wageningen University \& Research, Wageningen, The Netherlands. ${ }^{5}$ Department of Earth and Environmental Science, Macquarie University, Sydney, New South Wales, Australia. ${ }^{6}$ Red Cross Red Crescent Climate Centre, The Hague, The Netherlands. ${ }^{7}$ International Research Institute for Climate and Society, Columbia University, New York, NY, USA.

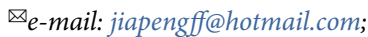
t.wang@utwente.nl

Published online: 13 February 2020 https://doi.org/10.1038/s41477-020-0598-2

References

1. Lewis, S. L., Wheeler, C. E., Mitchard, E. T. A. \& Koch, A. Nature 568, 25-28 (2019)

2. Bastin, J. F. et al. Science 365, 76-79 (2019).

3. Maier, H. et al. Br. J. Dermatol. 149, 990-997 (2003).

4. Spiegel, W., Maier, H. \& Maier, M. Lancet 363, 1438 (2004).

5. Toxic processionary caterpillar plague spreads across Europe. BBC https://www.bbc.co.uk/news/world-europe-48880468 (2019).

6. Ding, D. et al. Lancet 388, 1311-1324 (2016)

7. Jia, P. Lancet Planet. Health 3, e57-e59 (2019).

8. Distribution of Atlas moths. National Database Flora and Fauna https://www.verspreidingsatlas.nl/I0174 (2020).

9. Willis, K. J. \& Petrokofsky, G. Science 356, 374-376 (2017). 10. Is the Sneez'n Season Getting Longer and Stronger? Canberra Pollen Count and Forecast https://www.canberrapollen.com.au/ news-events/is-the-sneezn-season-getting-longer-and-stronger/ (2019).

11. Heylen, D. et al. Sci. Total Environ. 670, 941-949 (2019).

\section{Acknowledgements}

This work is supported by the research grant from the State Key Laboratory of Urban and Regional Ecology of China (grant no. SKLURE2018-2-5). P.J. thanks the Netherlands Organization for Scientific Research, the Royal Netherlands Academy of Arts and Sciences, the Chinese Center for

Disease Control and Prevention and the West China School of Public Health in Sichuan University for funding the ISLE and supporting the ISLE's research activities.

\section{Author contributions}

T.W. and P.J. conceived the idea and collected the data. P.J. and T.W. drafted the manuscript. A.V.V., M.V.A. and A.S. provided critical comments and revised the manuscript.

\section{Competing interests}

The authors declare no competing interests. 